\title{
IdeAs
}

Idées d'Amériques

$17 \mid 2021$

Villes et culture dans les Amériques

\section{Le rôle de la culture et des industries créatives dans la reconversion territoriale de Detroit : la production d'un espace adapté au capitalisme cognitif-culturel}

Cognitive-Cultural Capitalism and the Emergence of Greater Downtown Detroit El papel de la cultura y de las industrias creativas en la reconversión territorial de Detroit: la producción de un espacio adaptado al capitalismo cognitivocultural.

\section{Simon Renoir}

\section{OpenEdition Journals}

Édition électronique

URL : https://journals.openedition.org/ideas/10429

DOI : 10.4000/ideas. 10429

ISSN : 1950-5701

Éditeur

Institut des Amériques

\section{Référence électronique}

Simon Renoir, «Le rôle de la culture et des industries créatives dans la reconversion territoriale de Detroit : la production d'un espace adapté au capitalisme cognitif-culturel », IdeAs [En ligne], 17 | 2021, mis en ligne le 01 mars 2021, consulté le 07 juin 2021. URL : http://journals.openedition.org/ideas/ 10429 ; DOI : https://doi.org/10.4000/ideas.10429

Ce document a été généré automatiquement le 7 juin 2021.

\section{(c) (†) $\ominus$}

IdeAs - Idées d'Amériques est mis à disposition selon les termes de la licence Creative Commons Attribution - Pas d'Utilisation Commerciale - Pas de Modification 4.0 International. 
Le rôle de la culture et des industries créatives dans la reconversion territoriale de Detroit : la production d'un espace adapté au capitalisme cognitifculturel

\author{
Cognitive-Cultural Capitalism and the Emergence of Greater Downtown Detroit \\ El papel de la cultura y de las industrias creativas en la reconversión territorial \\ de Detroit: la producción de un espacio adaptado al capitalismo cognitivo- \\ cultural.
}

Simon Renoir

\title{
Introduction
}

En juillet 2013, la ville de Detroit déclarait faillite au terme d'un long déclin économique et budgétaire. En décembre 2015, elle entrait dans le réseau Unesco des «Villes du Design» et officialisait ainsi une politique de reconversion en «Ville Créative ». En ce début des années 2010, les récits médiatiques sur Detroit se sont inversés : de l'archétype de la ville en déclin, elle est devenue le symbole de la ville en plein renouveau, grâce notamment à la culture, la créativité et la gentrification (Doucet, B. 2020). Cet article traite du rôle de la culture et de la créativité dans le renouveau supposé de Detroit en «ville créative». Une ville en faillite et en décroissance (Béal, V. et al., 2019) peut-elle connaitre aussi rapidement un tel renouveau? Quelle serait la nature de ce renouveau en « ville créative »? 
Répondre à ces questions nécessite d'abord quelques précisions sur le contexte de Detroit et sur la notion de ville créative. Avant tout, Detroit est avec Milwaukee l'aire métropolitaine la plus ségréguée des États-Unis (Thomas, J. M. et H. Bekkering, 2015 ; Seymour, E. et J. Akers, 2019) (fig. 1). À l'instar d'autres villes industrielles du nord-est des États-Unis mais à un degré plus extrême, la métropole de Detroit incarne l'histoire raciale et urbaine du pays au $\mathrm{xx}^{\mathrm{e}}$ siècle (Sugrue, T., 1996). Il en a résulté que de larges portions de la ville-centre ( inner city ») sont devenues un « hyperghetto » (au sens de Wacquant, L. 2005), tandis que les villes des «suburbs " sont très majoritairement composées de classes moyennes et aisées blanches.

Fig. 1 : Répartition de la population par origine ethnique et raciale dans l'aire métropolitaine de Detroit

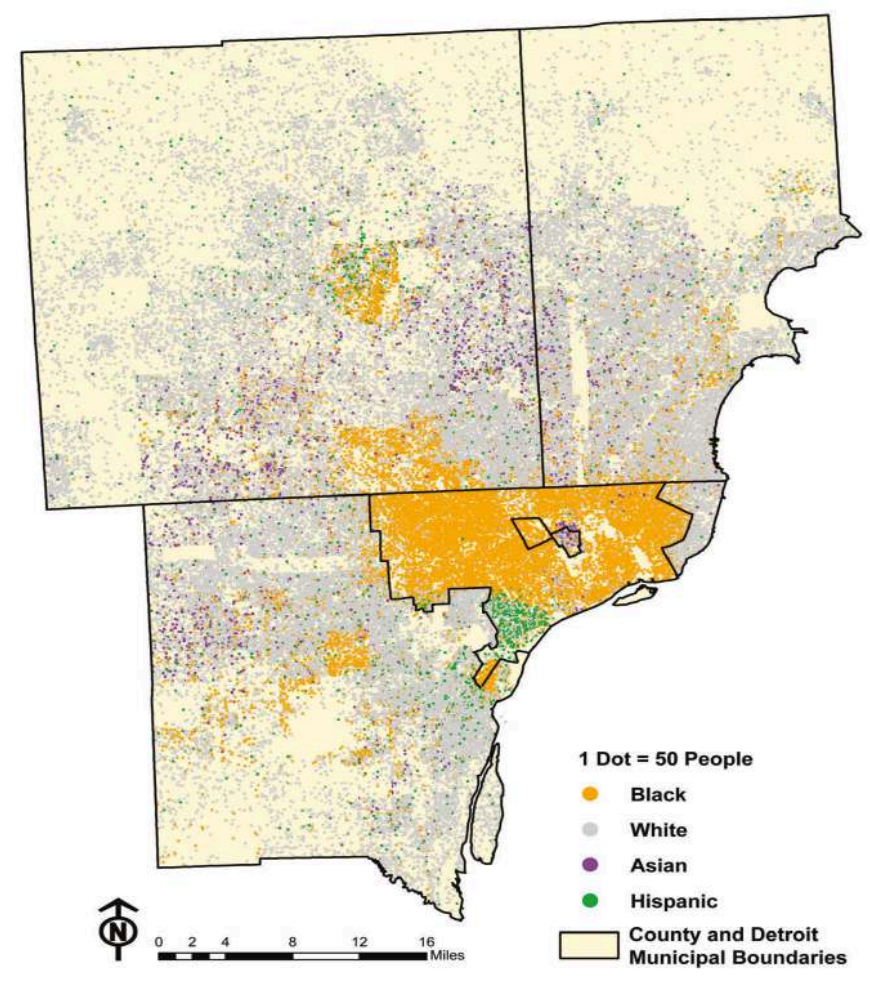

Thomas et Bekkering, 2015

Dans ce contexte, les reconfigurations actuelles s'inscrivent dans un régime d'« urbanisme d'austérité » (Briche, H. 2016). L'action publique y est de plus en plus guidée par les impératifs du marché («market-based governing ») et fragmentée entre, d'un côté, des zones et quartiers riches ou relativement prospères qui continuent à recevoir des investissements et des services publics et de l'autre, des quartiers pauvres (souvent périphériques) où les habitants doivent assurer eux-mêmes certains services publics essentiels (Kinder, K. 2016). Cela aboutit à la formation d'une ville divisée entre une zone centrale appelée "7.2 Square Miles» ou "Greater Downtown» (désormais GD) (Doucet, B. et E. Smit, 2016) et des quartiers périphériques. Le renouveau en «Ville Créative » mis en avant dans les récits médiatiques ne concerne que la zone du GD, qui ne représente que $5 \%$ de la superficie et de la population de la ville (voir fig. 2 et 3 ). 
Fig. 2 : Le Greater Downtown dans la ville de Detroit

Greater Downtown Detroit

Square Miles: 7.2 so. Mil

Population: 36,550 people

Density: 5.076 People/SQ. Mt.

Per Capita Income: $\$ 20,21$

Hudson-Webber Foundation 2013

Fig. 3 : Les quartiers composants le Greater Downtown

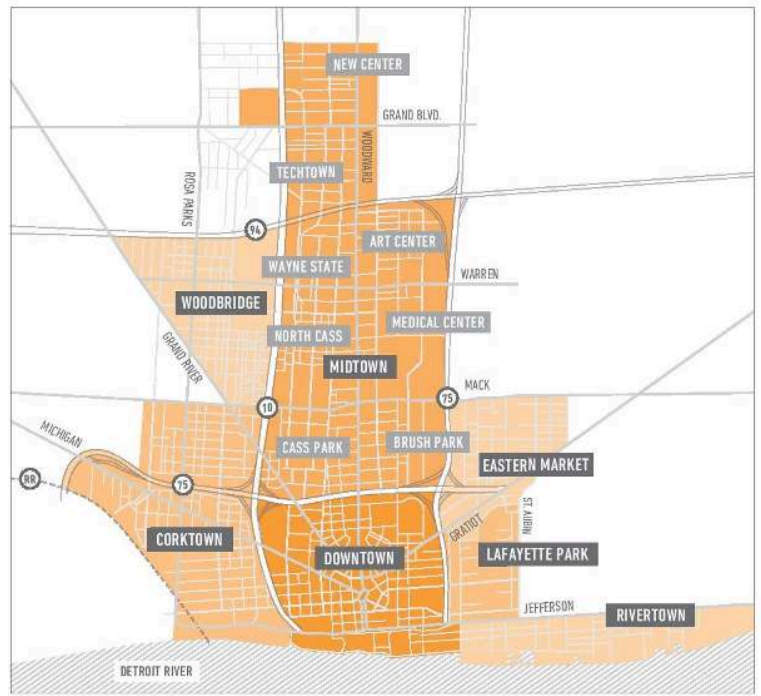

Hudson-Webber Foundation 2013

4 Pour ce qui concerne la notion de "ville créative ", nous nous appuyons sur l'analyse du géographe Allen J. Scott (voir Scott, A. J., 2014a et 2014b) qui propose de déconstruire la notion et de la remplacer par celle de « villes du capitalisme cognitif- 
culturel ». Selon lui, l'expression « ville créative » ne désigne qu'un trait caractéristique parmi d'autres dans la transition désormais achevée d'un régime d'accumulation fordiste du capitalisme à un régime d'accumulation cognitif-culturel. Quatre traits distinctifs caractérisent ce régime : 1) l'économie est de plus en plus orientée vers la production de biens et de services non standardisés tels les hautes technologies, la finance, les services à la personne et les industries culturelles et créatives ; 2) ces secteurs ont une tendance à la désintégration horizontale et verticale des entreprises qui se recomposent en réseaux de petites unités de production très spécialisées et complémentaires ayant une forte propension à s'agglomérer sur un même territoire (à ce sujet, voir Piore, M. et C. Sabel, 1984/1989 ; Cooke, P. et L. Lazzerretti, 2008) ; 3) une part de plus en plus importante de la valeur ajoutée dans ces secteurs provient de la singularité de leurs produits, laquelle est associée à une marque, un lieu ou une origine (ce point rejoint les travaux de Karpik, L., 2007, ou encore Boltanski, L. et A. Esquerre, 2017) et 4) l'expansion d'une main d'œuvre possédant un haut niveau de compétences cognitives et culturelles, qui correspond à peu près à la « classe créative » décrite par Richard Florida (2002).

5 Comme son nom l'indique, la culture occupe une place centrale dans ce nouveau régime $\mathrm{du}$ capitalisme et dans les reconfigurations socio-spatiales qui l'accompagnent. Précisons toutefois que notre intérêt pour la culture porte sur la façon dont, depuis une vingtaine d'années, la référence à la créativité et à ses variantes (industries, économie, ville, classe créatives) a transformé à la fois les catégories d'action publique et les significations traditionnellement associées au concept de culture.

Les expressions «industries créatives" et "ville créative » présentent toutes les caractéristiques de ce que certains (comme Desage, F. et J. Godard, 2005) ont proposé d'appeler des «mythes » en matière de politiques publiques et que d'autres nomment des «formules» discursives (Krieg-Planque, A., 2010). Le succès de ces formules vient de leur imprécision, laquelle permet d'agréger des acteurs sociaux très différents autour de projets communs (Garnham, N., 2005) et autorise chaque groupe d'acteurs à s'y référer et à y transposer ses propres attentes, représentations et intérêts (Desage, F. et J. Godard, op. cit.). De surcroît, formules et mythes dans le cadre de l'action publique sont mobilisés de manière rhétorique pour obtenir des crédits et tirent leur force de leur capacité à "mettre en récit » l'action publique et à la légitimer (ibid.; KriegPlanque, A., op. cit.).

7 Les travaux (notamment ceux issus de l'économie politique de la communication) qui ont étudié ce glissement des catégories installées de culture et d'industries culturelles vers les formules de la créativité tirent plusieurs conclusions. L'inclusion dans une même catégorie (les industries créatives) d'activités relevant des arts et de la culture, des industries culturelles et d'autres auparavant situées hors du secteur culturel comme le design et le software (ou, ponctuellement, d'autres activités intensives en technologie) présente un double avantage: d'une part, il permet à ce secteur dit "créatif " d'afficher un volume d'affaires et un taux de croissance exceptionnels, attribuables principalement au design et aux technologies; d'autre part, il permet à toutes ces activités de bénéficier du prestige associé au travail créateur des artistes (Garnham, N., op. cit. ; Tremblay, G., 2008). Ce dernier point participe d'un mouvement plus général de « culturisation de l'industrie » qui consiste en « l'attribution de qualités ou grandeurs culturelles et symboliques à des produits non culturels mais plus ou 
moins artificiellement investis d'une dimension créative » (Bouquillion, P. et al., 2013 : 8).

8 De plus, dans les projets des promoteurs de l'économie créative, les descriptions de la créativité se limitent à des facteurs d'amélioration des performances économiques (Bouquillion, P. et al., 2015). La référence à la notion de créativité contribue alors à une tendance à la conversion des artistes en entrepreneurs (ibid.) et les dispositifs d'aide à destination du secteur s'éloignent des politiques culturelles traditionnelles pour se rapprocher de politiques industrielles de soutien de l'innovation (Bouquillion P., 2012).

Toutes les références à la culture et aux industries créatives dans cet article renvoient à ces définitions. À partir de ces constats, nous pourrons nous demander quel rôle ont joué la culture et les industries créatives dans la transition de la ville de Detroit vers un régime d'accumulation cognitif-culturel? Avec quels effets ? Pour y répondre, nous faisons l'hypothèse que les formules de la créativité permettent de légitimer les diverses actions mises en œuvre dans ce cadre, lesquelles ont consisté principalement à revitaliser le "Greater Downtown » en le densifiant et en l'esthétisant, et à changer l'image de la ville pour l'associer au design (Renoir S., 2019).

10 Nous explorerons cette hypothèse en nous appuyant sur des données provenant d'une enquête de terrain réalisée au cours de trois séjours à Detroit (entre janvier 2015 et septembre 2017) dans le cadre d'une recherche doctorale. Cette enquête regroupe quarante entretiens semi-directifs avec des responsables d'organisations locales actives dans le développement économique et/ou culturel, de fondations philanthropiques, d'entreprises du secteur créatif et d'associations et collectifs d'artistes, ainsi que des données d'observation dans les espaces urbains, dans les lieux culturels locaux et lors d'événements (Detroit Design Festival 2015 et 2017, Creative Economy Policy Summit et Biennale du Design de Saint-Etienne en 2017). Nous complétons ces données par une analyse des discours et des stratégies des acteurs locaux à partir d'un corpus d'articles de presse et de rapports institutionnels.

11 Un premier point traitera de la référence à la créativité comme point de rassemblement dans le contexte plus large des projets de revitalisation des années 2010. Un deuxième point examinera le rôle de la culture et des industries créatives dans les reconfigurations sociales et spatiales de la ville.

\section{La référence à la créativité comme point de rassemblement des projets de revitalisation urbaine}

12 À l'origine de la récente reconversion territoriale de Detroit se trouve un plan de relance intitulé "Road to Renaissance", établi en 2006 sous l'égide de Detroit Renaissance Inc. (devenu en 2009 Business Leaders for Michigan - BLM), principal groupe d'influence de la région et acteur central des différents régimes urbains locaux depuis 1970 (Hall L. et M. F. Hall, 1993; Neill W., 1995). Ce plan fait référence à l'économie créative en tant que secteur d'avenir devant permettre de réorienter l'économie locale et d'amoindrir la dépendance de l'industrie automobile (Detroit Renaissance, 2006). À partir de 2009, ce plan donne naissance à deux organisations. Créée par un partenariat entre BLM et le College for Creative Studies (principale école de design et arts appliqués de la ville), le «Detroit Creative Corridor Center » (DC3) était une agence spécialisée dans le développement des industries créatives qui avait 
pour mission d'établir puis d'animer le "Creative Corridor " (sorte de district créatif dont il sera question plus loin). En mars 2018, le DC3, rebaptisé « Design Core Detroit ", a recentré sa mission sur la gestion du label Unesco « Ville du Design ». Issue d'un effort collectif de douze fondations philanthropiques locales et nationales, la « New Economy Initiative " (NEI) structure un "écosystème» de soutien à l'entrepreneuriat et à l'innovation par le financement de multiples acteurs intermédiaires: incubateurs et accélérateurs (dont le DC3 et le plus important de la ville, TechTown), organismes de formation (Build Institute) et organismes de crédit (Invest Detroit).

Dans le même temps, d'autres institutions et des acteurs privés déploient des plans de relance et d'investissement. La Ville de Detroit dévoile en décembre 2012 son « Detroit Strategic Framework Plan » (Detroit Future City, 2012) résultant d'un partenariat avec des fondations comme la Kresge Foundation. Imaginant la ville à l'horizon 2050, ce projet urbanistique est ambitieux. Il assume le déclin de la ville et préconise de transformer les zones à faible densité en quartiers verts comprenant des zones de production (agriculture, pisciculture, bois et énergies renouvelables). Entre ces espaces semi-ruraux, sept quartiers d'activités sont ciblés pour le redéveloppement, dont cinq devraient se spécialiser dans les industries numériques et créatives.

De son côté, la Kresge Foundation s'est quasiment substituée à la puissance publique en élaborant une véritable stratégie urbaine touchant à tous les domaines (transport, avec le financement de la construction d'un tramway, santé, éducation/formation et sécurité), dont un pan important est consacré au financement des arts et de la culture, et au soutien au secteur créatif loué pour sa capacité de " résilience » en temps de crise (Kresge Foundation, 2012).

Deux hommes d'affaires milliardaires investissent dans leurs propres projets qui s'inscrivent dans le modèle socio-spatial du capitalisme cognitif-culturel. Entre 2010 et 2015, Dan Gilbert, propriétaire de la firme de prêts immobiliers en ligne Quicken Loans, a investi environ 1,7 milliards de dollars au centre-ville. Il a acheté et réaménagé environ soixante-dix gratte-ciels dans lesquels il a attiré douze mille cinq cents travailleurs (Briche H., op. cit.), dont les quatre mille employés de son siège social, Bizdom, un incubateur de start-ups du numérique auparavant situé à Livonia dans les suburbs et des bureaux qu'il loue à des firmes numériques ou créatives. À titre d'exemple, le M@dison Building accueille les bureaux locaux de Twitter, des start-ups du numérique comme Detroit Labs ou encore Skidmore Studio (une agence de communication et de design).

Pour sa part, la famille Ilitch, propriétaire de la chaîne de fast-food Little Caesars, a créé «The District Detroit » qui est organisé autour de la construction d'une nouvelle salle de sport et de spectacle, The Little Caesars Arena, entourée de logements, d'hôtels, d'espaces commerciaux et de divertissement, et située dans une zone adjacente aux stades et aux salles de spectacle du «Theatre District » qui appartiennent aussi à la famille Ilitch. Le coût de ce district est estimé à environ huit cent soixante millions de dollars. L'État du Michigan s'est fortement engagé dans ce projet en apportant trois cent vingt millions de dollars via des émissions d'obligations, intervention justifiée au nom de la culture dans la mesure où elle favorise un mode de vie urbain qui répond aux attentes de la "classe créative » (voir l'entretien avec Mike Finney, ex-directeur de la Michigan Economic Development Corporation, 06/11/2015). De même la ville de Detroit a vendu à la famille Ilitch trente-neuf parcelles dont elle était propriétaire pour un 
dollar symbolique alors que leur valeur était estimée à trois millions de dollars (Briche, H., op. cit.)

17 En dépit de leur diversité, les projets mis en œuvre par ces acteurs poursuivent les mêmes objectifs : la revitalisation du centre-ville et l'attraction de la " classe créative ". En cela, la reconversion actuelle marque l'aboutissement d'un long processus de conciliation entre les deux régimes urbains qui coexistent localement et sont en concurrence depuis les années 1980 (Orr, M. et G. Stoker, 1994). Ainsi, il semble que les formules faisant référence à la créativité, devenues des mythes de l'action publique aient été des «fétiches rassembleurs » (Desage, F. et J. Godard, op. cit.) de ces initiatives dispersées, favorisant leur conciliation vers des objectifs similaires ainsi que leur légitimation. Outre ce rôle discursif et idéel, la culture et les industries créatives jouent un rôle plus opérationnel dans la reconfiguration sociale et spatiale de la ville.

\section{Le rôle de la culture et des industries créatives dans la reconfiguration de la ville}

Nous identifions deux transformations socioculturelles et sociospatiales principales dans lesquelles la culture et les industries créatives ont joué un rôle essentiel: le changement d'image de la ville et la production d'un espace dense et esthétisé au centre-ville, renommé « Greater Downtown » (GD).

\section{«A Tale of Two Cities » : la ville divisée par les récits}

L'image d'un territoire est notamment façonnée par les récits et représentations médiatiques qui le dépeignent (Noyer, J. et al, 2013). Parmi ceux qui racontent le renouveau de Detroit, nous avons identifié trois axes principaux: le "Nouveau Brooklyn » de l'avant-garde artistique et de la culture "off» (au sens où l'entend Vivant, E., 2009), "Gilbertville », qui fait de Dan Gilbert le héros et le sauveur de Detroit, et le «Design Made In Detroit " autour du label Unesco «Ville du Design » obtenu par l'action du DC3. Nous ne détaillerons que ce dernier. D'origine institutionnel, il se situe dans le registre du « city branding »et vise à donner à la ville une nouvelle image de marque en faisant du design sa " marque Territoire » (HoullierGuibert, C.-E., 2017). Il est mis en œuvre par le DC3 qui communique à partir de 2010 autour d'événements qu'il a créés, le DrinksxDesign (dîners apéritifs mensuels organisés dans des studios de design) et le Detroit Design Festival. Puis le DC3 obtient le label Unesco « Ville du Design » en décembre 2015.

Le processus de "rebranding " a eu des effets sur les entreprises du secteur, plusieurs d'entre elles (comme Shinola, Skidmore Studio et Sundberg Ferar) nous ont affirmé avoir été en mesure d'augmenter leurs prix grâce à la nouvelle marque Territoire qu'elles contribuent d'ailleurs à construire et qui associe Detroit à un certain design et à des valeurs spécifiques. Par exemple, les marques Shinola et Detroit vs. Everybody mobilisent l'imaginaire d'une ville qui lutte contre le déclin et n'abandonne jamais (voir l'entretien avec Jacques Panis, Shinola, 30/11/2015). En novembre 2015, Shinola (en partenariat avec le Muhammad Ali Center basé à Louisville, dans le Kentucky) a produit des éditions limitées de montres au design inspiré de l'esprit combattif de Mohammed Ali et en a assuré la promotion en organisant une exposition dédiée au boxeur dans sa boutique. Ces procédés sont par ailleurs représentatifs de la tendance à 
la "culturisation de l'industrie " qui se déploie dans les industries créatives (Bouquillion, P. et al., op. cit.) et sont une première manifestation de la transition vers un capitalisme cognitif-culturel à travers la commercialisation de produits non standardisés et la singularité associée à une marque et un lieu.

De surcroît, ces trois axes s'assemblent pour créer un récit unique. Celui-ci compose l'image d'un «nouveau Detroit» créatif et innovant, concentré sur la zone géographique restreinte $d u G D$. Le récit cible les travailleurs créatifs et les jeunes cadres en leur promettant un mode de vie marqué par l'esprit d'aventure et l'aspiration à la liberté grâce à la culture et à l'absence de barrières à l'entrée (les prix de l'immobilier sont très bas), dans une ville décrite comme la "nouvelle frontière " (" new frontier ») et comparée à l'Ouest sauvage où tout est à réinventer (Renoir, S. 2018). Dans la mesure où il convoque le mythe américain de la « Frontier » qui puise ses origines dans l'imaginaire colonial de la conquête de l'Ouest (Slotkin, R. 1998), ce récit est teinté d'un esprit néocolonialiste (Safransky, S. 2014) qui a parfois été associé au phénomène de gentrification dans les « inner cities » américaines (Smith, N. 1996).

En outre, ce récit « invisibilise » la culture africaine-américaine, pourtant largement majoritaire à Detroit. Par exemple, l'absence de référence musicale dans ces nouveaux récits et dans la stratégie d'intégration au réseau Unesco des "Villes Créatives » peut étonner (Renoir S., 2019) et suscite des critiques (entretien avec Tiff Massey, artiste, 11/02/2016; entretien avec Cornelius Harris, manager du label Underground Resistance, 28/09/2017). Ainsi, un conflit culturel et identitaire s'est installé entre un «nouveau Detroit» concentré au centre-ville et associé à la culture des classes moyennes et aisées blanches et un «ancien Detroit» de culture ouvrière et essentiellement africaine-américaine. Ce conflit, fréquemment désigné par l'expression "A Tale of Two Cities », a pris une telle ampleur qu'à l'été 2017, Mike Duggan, le maire de la ville, a créé une fonction inédite de "Chief Storyteller». Le poste a été attribué à Aaron Foley, journaliste et leader d'opinion de la communauté noire, avec pour mission d'apaiser la fracture identitaire et de faire émerger un récit alternatif mettant en avant la culture des quartiers et de la communauté africaine-américaine (entretien avec Aaron Foley, 29/09/2017). Or, après trois années de fonctionnement, il semble que ce service n'ait pas réussi à faire émerger un récit alternatif.

\section{La production spatiale du Greater Downtown}

Aux transformations sur le plan narratif correspondent des transformations sur le plan spatial. Celles-ci se produisent par trois types d'opération qui font intervenir à des degrés variés la culture et les industries créatives.

\section{La création d'une « ville dans la ville »}

L'idée de créer un espace dense et homogène au centre-ville de Detroit pour attirer le capital humain remonte aux années 1980. On en relève déjà la trace dans le Detroit Strategic Planning Project, un plan de relance qui marquait le début de la transition vers un régime urbain de l'attraction du capital humain (Orr, M. et G. Stoker, op. cit.), dont les recommandations n'ont jamais vraiment été suivies d'effets. À l'époque, la zone identifiée comme une "ville dans la ville» («a town within the city ») comprenait les quartiers de Midtown et du New Center et elle devait concentrer logements, commerces, bureaux et lieux de loisirs (Detroit Renaissance, 1987). 

GD ont été réellement conçus comme un ensemble homogène. Sous l'impulsion de la Hudson-Webber Foundation, un ensemble d'acteurs (parmi lesquels la Detroit Economic Growth Corporation de la ville de Detroit et des organisations de développement de quartiers comme Midtown Detroit Inc., Downtown Development Partnership, Detroit Riverfront Conservancy ou Eastern Market Corporation) vont s'atteler à l'élaboration et à la promotion de ce nouvel espace. En 2013 (puis en 2015), ils publient un rapport intitulé «7.2 Square Miles Report» qui popularise les expressions "Greater Downtown » et " 7.2 Square Miles ", et contribue à façonner l'existence d'un espace homogène dans l'imaginaire collectif (Doucet, B. et E. Smit, op. cit.)

Dans le même temps, ces acteurs entreprennent diverses opérations de régénération esthétique des espaces publics (Côté, J.-F. et M. Pugliese, 2008), typiques d'un aménagement de la ville par la culture (Gravari-Barbas, M. 2013) : réaménagement des parcs, voies vertes, quais et fronts d'eau, création de pistes cyclables. Ces opérations sont mises en place selon une démarche de "creative placemaking ", qui correspond à la mise en place de partenariats entre divers acteurs (publics, privés et associatifs) provenant de divers secteurs dont au moins un doit appartenir au secteur culturel (Markusen, A. et A. Gadwa, 2010). Omniprésente dans les discours sur la transformation urbaine et le mouvement des "villes créatives" aux États-Unis, cette notion a fait l'objet de nombreuses critiques pour ses effets d'homogénéisation de l'espace et de la culture (Zitcer, A., 2018). La Dequindre Cut (majoritairement financée par la HudsonWebber Foundation), une ancienne voie ferrée reconvertie en voie verte ouverte aux piétons et aux cyclistes, et embellie par de nombreuses œuvres commandées à des artistes issus du Street Art, en constitue l'exemple le plus emblématique (fig. 4).

Fig. 4 : La Dequindre Cut

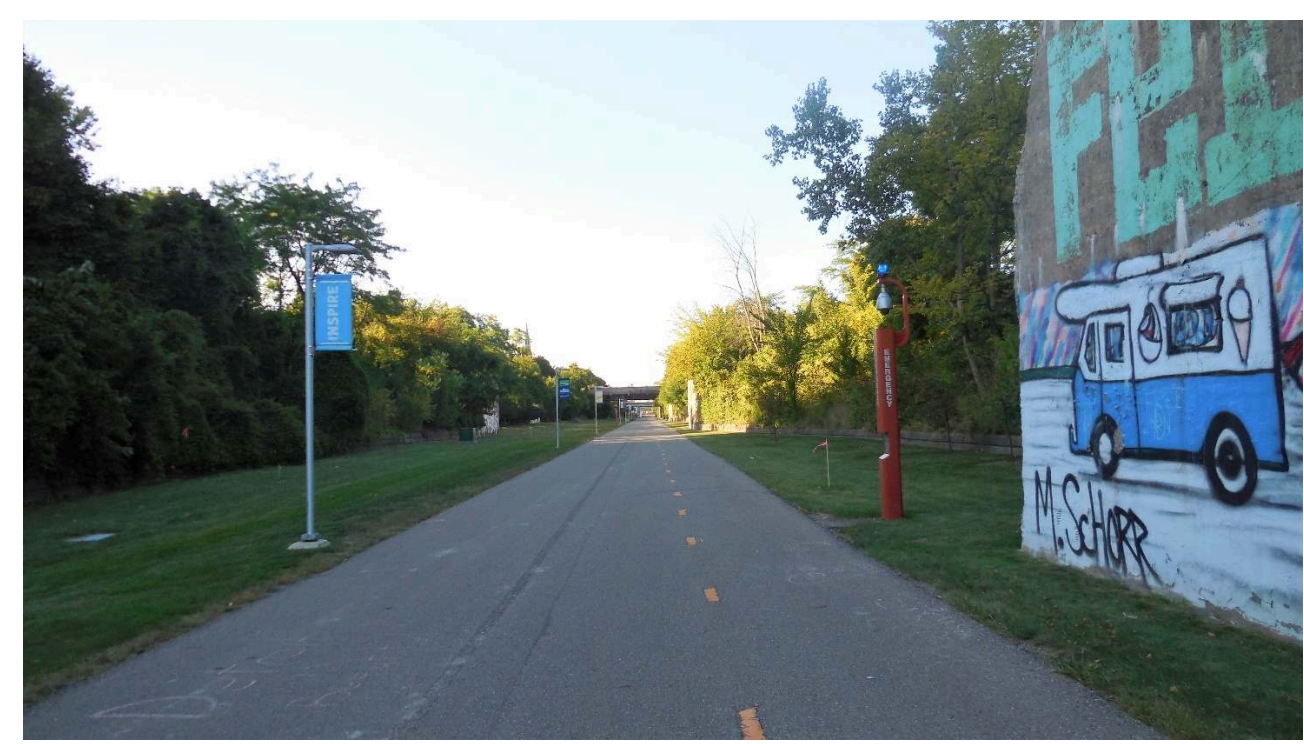

Photo de l'auteur (septembre 2015)

Cette démarche de "creative placemaking " s'attache autant à embellir les espaces par l'intervention de l'art qu'à les animer, en y organisant notamment des événements culturels. Depuis 2010, de nombreux événements sponsorisés et financés par ces mêmes 
acteurs ont ainsi vu le jour à l'intérieur du GD comme D:Lectricity (festival de lumière et du numérique, Midtown Detroit Inc.), Murals in the Market (festival de Street Art organisé par la Eastern Market Corporation), Open Streets Detroit (Downtown Development Partnership) et Detroit Design Festival (organisé par le DC3 en partenariat avec plusieurs de ces acteurs). Ces opérations, qui correspondent à ce qu'Allen Scott (op. cit) appelle une «intensification d'un aménagement du sol esthétisé », sont les instruments d'attraction de prédilection d'une main d'œuvre hautement qualifiée.

\section{Le découpage en districts du centre-ville}

En plus de la création et de la promotion du GD, de multiples districts dits " culturels " ou «créatifs » ont vu le jour. Tous se situent à l'intérieur du GD et leurs périmètres peuvent se superposer. On peut ainsi recenser The Innovation District, The District Detroit, The Paradise Valley Cultural \& Entertainment District et The Creative Corridor.

En 2010, le DC3 entame la mise en place du Creative Corridor. Sa première mission consiste à attirer des entreprises et des emplois créatifs à l'intérieur de cette zone géographique. La création d'un Creative Corridor Incentive Fund, un fonds qui offre des incitations financières aux entreprises désirant installer leur activité à l'intérieur du Creative Corridor, doit aider à accomplir cet objectif. Le fonds n'a toutefois pas été géré par le DC3, mais par la DEGC. Son enveloppe budgétaire, d'un montant de 2,5 millions de dollars provenait intégralement d'une dotation de la NEI. DEGC et NEI ont défini les critères d'obtention des financements en établissant la liste de dix-sept filières d'activités "créatives » éligibles appartenant aux secteurs de la bio-ingénierie, des technologies de l'information, des médias numériques, ou du spectacle vivant (entretien avec Mark Denson, DEGC, 11/04/2016).

0 La moitié du budget a été attribuée rapidement et « de façon opportuniste » en quatre subventions d'environ trois cent mille dollars chacune (entretien avec Olga Stella, exdirectrice adjointe du DEGC, puis directrice du DC3, 15/03/2016), dont trois ont pour vocation d'inciter des entreprises à relocaliser leur siège social au centre-ville de Detroit: GalaxE Solutions (informatique), Lowe Campbell Ewald (agence de communication et de publicité) et Skidmore Studio (agence de communication et de design). L'existence d'un « effet d'aubaine » de ces incitations pour ces entreprises nous a été confirmée par le PDG de la plus petite d'entre elles (entretien avec Tim Smith, PDG Skidmore Studio, vingt-cinq employés, 16/02/2016). La quatrième a été attribué à Bedrock, l'une des filiales de Dan Gilbert, pour la réhabilitation du M@dison Building en immeuble de bureaux destinés à héberger diverses entreprises des industries créatives (dont Skidmore Studio) ou du numérique.

La création de districts, et plus généralement la production d'un centre-ville dense et animé, répond à la nécessité de regrouper les entreprises du capitalisme cognitifculturel et leurs travailleurs au sein d'un espace restreint afin qu'elles puissent bénéficier d'effets d'agglomération (Piore, M. et C. Sabel, op. cit.). Ceux-ci favorisent la créativité et l'innovation. Au sujet des facteurs favorisant la créativité, Allen Scott (op. cit. : 569), en s'appuyant sur divers travaux, conclut que la créativité s'acquiert dans une large mesure à travers l'éducation, l'apprentissage de savoirs et savoir-faire et leur mise en pratique, ainsi que par la socialisation informelle. Lorsque des individus sont insérés dans des réseaux transactionnels denses, ils échangent davantage d'informations et peuvent explorer davantage de possibilités, ce qui accroît la créativité de tous, selon un phénomène parfois désigné par le terme de " pollinisation » 
(Moulier-Boutang, Y. 2014). C'est principalement pour cette raison que les secteurs économiques clés du capitalisme cognitif-culturel, qui reposent sur l'information, la connaissance et la créativité, s'inspirent du mode d'organisation qui prévaut dans les mondes des arts et de la culture, lequel est structuré en "mode projet» avec une spécialisation souple et une organisation extensive favorisant la coopération entre petites firmes (Menger, P-M. 2002). À cet égard, le découpage en districts du centreville dénote une vision de la culture, redéfinie par la référence à la créativité, orientée vers l'innovation et la performance économique.

\section{La réhabilitation d'espaces vacants : une resémantisation de l'espace}

Enfin, de nombreux espaces vacants ont été réhabilités en lieux de production et de diffusion artistique ou en lieux de consommation. Dans un premier temps, ces réhabilitations ont principalement été faites en-dehors des institutions, par des artistes ou des entrepreneurs, qui financent les travaux avec leurs fonds propres ou bricolent eux-mêmes le réaménagement de leur espace selon une éthique DIY (« do it yourself ») très répandue à Detroit (Paddeu, F., 2012; K. Kinder, 2016). Ces initiatives fleurissent en particulier dans les anciens quartiers d'industrie légère et de manufacture limitrophes du centre-ville (comme Eastern Market, Corktown et New Center), qui regorgent de friches à reconvertir.

Après ce temps d'appropriation des espaces vacants, un " temps de consolidation " (Ambrosino, C. et L. Andres, 2008) via l'intervention des pouvoirs publics a vu le jour. Plusieurs programmes d'aide (comme le NEIdeas piloté par la NEI ou le Retail Boot Camp de TechTown) ont été créés afin de soutenir ces projets dont le plus emblématique est Motor City Match, porté par la DEGC. Depuis 2012 ce dispositif met en relation des propriétaires d'espaces vacants, des commerçants à la recherche d'un espace et des artistes, designers ou architectes missionnés pour redessiner l'espace. L'accent était d'abord mis sur l'aspect artistique et créatif à travers la création de boutiques et galeries d'art éphémères, avec pour objectif d'occuper les espaces vides à moindre frais. Depuis 2015, le programme a évolué vers l'aide financière à la réhabilitation d'espaces en distribuant cinq cent mille dollars par trimestre à une dizaine de projets. Ainsi, Assemble Sound, une entreprise musicale a obtenu le premier prix (d'un montant de cent mille dollars) au premier trimestre 2016 pour pouvoir réaménager l'église qui accueille ses locaux (fig. 5). 


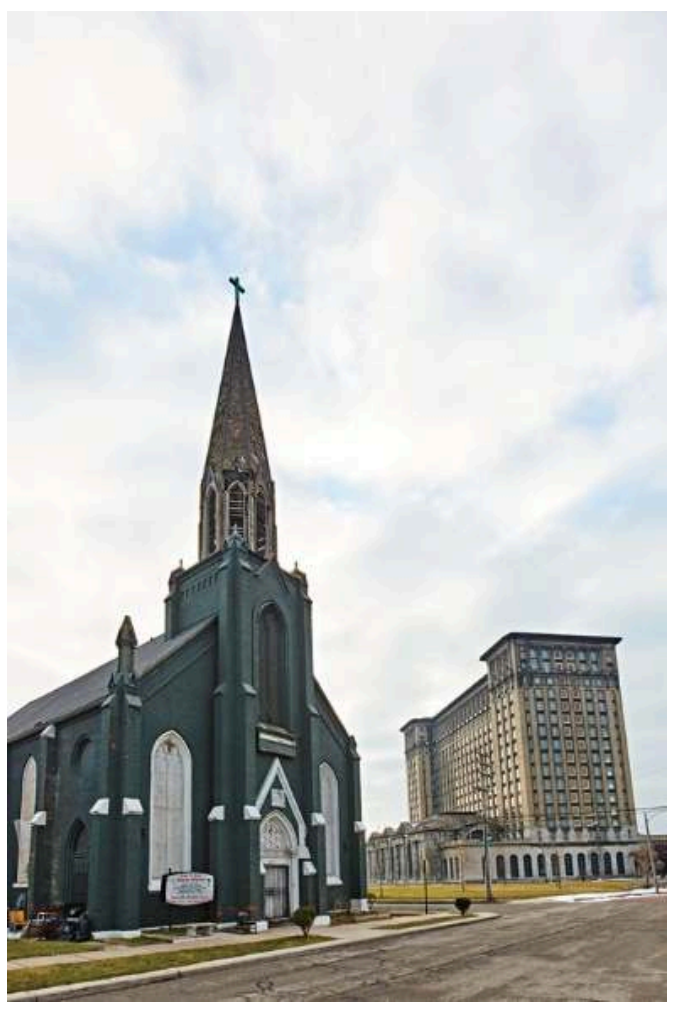

Matthew LaVere photography

Les lieux reconvertis accueillent des activités artistiques (Red Bull House of Art), des industries créatives (studios de design) ou des espaces de travail partagé (Omnicorp, Ponyride). Le plus souvent, ils accueillent des espaces commerciaux ou «boutiques " (en anglais), soit des commerces de nouvelle génération doté d'une ambiance " design » et ciblent une clientèle aisée à la recherche de singularité, de distinction sociale et d'authenticité (Zukin, S. et al., 2009). Dans tous les cas, ces lieux reconvertis peuvent prétendre entrer dans le champ de la créativité, voire de la culture. Le geste de reconversion assure leur caractère créatif et la conservation des traces du passé industriel leur confère une authenticité propre aux lieux de patrimoine (Renoir, S. 2018).

Ces nombreuses reconversions de friches (plutôt concentrées dans le GD, même si ces opérations sont pratiquées dans toute la ville) participent de la resémantisation du territoire (Reitel, B. et F. Moullé, 2015 ; Mortelette, C., 2019) à travers un changement de fonction et de forme qui conduit plus profondément à un renouvellement du sens de l'espace urbain. Par le biais de ces opérations créatives, la ville réalise la transition, tant sur le plan fonctionnel que symbolique, d'un espace organisé selon les principes du capitalisme fordiste (production de masse et fonctionnalisme) à un espace organisé selon ceux du capitalisme cognitif culturel (consommation distinctive et de singularité, « mass customization »).

\section{Alternatives et perspectives}

Notre analyse du rôle de la culture et des industries créatives dans les reconfigurations sociales et spatiales de la ville serait incomplète si elle omettait de mentionner d'autres 
alternatives, certes moins visibles et disposant de moyens limités, mais bien réelles. Il existe à Detroit d'importants mouvements « grassroots » de contestation des politiques d'austérité et des projets dominants de revitalisation du centre-ville. Notamment structurés autour des jardins ouvriers partagés et de revendications pour la justice environnementale et alimentaire (Paddeu, F. 2015), ces mouvements développent des pratiques économiques alternatives, dans la mesure où il s'agit de pratiques " conscientes orientées vers une économie fondée sur la valeur d'usage " (Castells cité par Paddeu, F. 2012). Sur le plan culturel, ces mouvements luttent pour la reconnaissance de la culture africaine-américaine et contre son "invisibilisation", voire son «blanchiment » comme c'est le cas pour la musique techno (Benabdellah, A. 2020).

Par exemple, dans le quartier du North End, un projet alternatif de développement a vu le jour. Plusieurs acteurs locaux y coopèrent autour de l'agence de design Akoaki, d'une ferme urbaine (Oakland Avenue Urban Farm) et d'un réseau de musiciens, d'artistes et de résidents (O.N.E. Mile, Detroit Afrikan Music Institute, Detroit Funk Orkestra). Ils mettent en œuvre un projet commun de développement économique et de préservation de la culture afro-américaine à travers des dispositifs innovants: constitution de " communs " par l'achat et le partage de terrains au sein de la ferme, expérimentation de monnaies locales et de marchés locaux de troc, créations diverses inspirées de l'afrofuturisme (musique, scénographie, installations d'art et de design, arts plastiques, voir fig. 6).

Fig. 6 : Le Mothership (qui sert de stand DJ) dans le garage ONE Mile, quartier North End

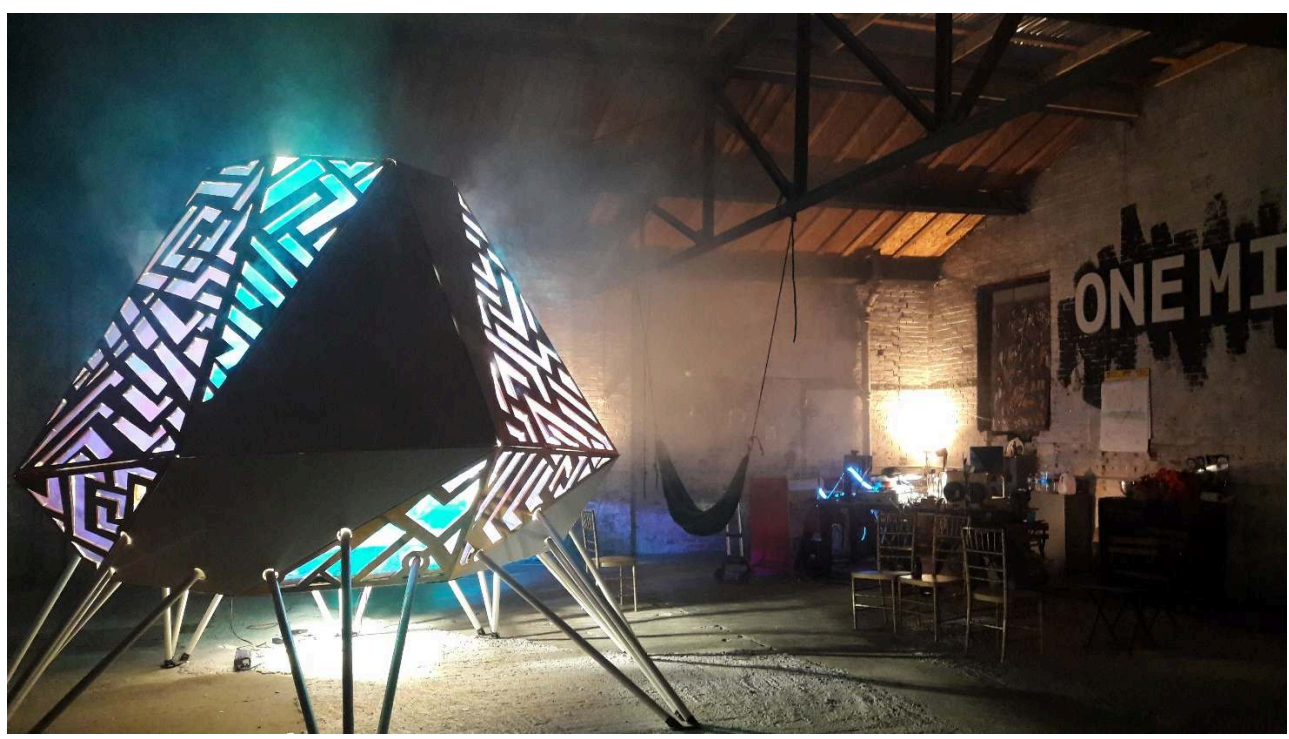

Photo de l'auteur (septembre 2017)

Plus généralement, ces acteurs critiquent la médiatisation nouvelle de pratiques anciennes et le fait que l'essentiel de la valeur générée par ces pratiques culturelles est capté par les acteurs du Greater Downtown et par les nouveaux arrivants artistes et entrepreneurs (entretien avec Tiff Massey, artiste, 11/02/2016; entretien avec Bryce Detroit, One Mile, artiste, responsable de projets culturels, 26/09/2017). C'est en particulier le cas du Street Art et des pratiques DIY de bricolage réinvestis dans l'art. Auparavant passée sous silence, la médiatisation soudaine de ces pratiques est vécue 
comme une appropriation de la culture populaire locale par les classes moyennes, porteuse de violence symbolique (Gravari-Barbas, M. et F. Ripoll, 2010).

Ces alternatives pourront-elles suffisamment se faire entendre pour être en mesure de proposer et de pérenniser un véritable projet urbain alternatif ? Rien n'est moins sûr. La tendance générale semble plutôt aller vers une intensification de la gentrification, déjà en cours au centre-ville (Doucet, B. op. cit.; Mah, J. 2020). Les reconfigurations symboliques et spatiales $\mathrm{du}$ GD ouvrent en effet sur une nouvelle vague d'investissements qu'illustre le projet de Ford Motor Company de créer un grand campus industriel dans le quartier de Corktown. Depuis 2017, le constructeur automobile a reconverti plusieurs bâtiments industriels et, plus encore, il a racheté l'ancienne gare centrale, la friche la plus emblématique de Detroit (fig. 7). Il y entreprend une vaste opération de reconversion en lieu multifonctionnel qui accueillera des espaces de travail pour ses équipes en charge de la R\&D, de la commercialisation et de la promotion de ses futurs véhicules autonomes, mais aussi des lieux de consommation et de socialisation (boutiques, bars et restaurants). Dans une interview donnée en juin 2018 pour Detroit Free Press Bill Ford Jr., l'actuel PDG de Ford, justifiait cet investissement dont le coût est estimé à huit cents millions de dollars : "we're on a war for talent and if you think about the great tech companies that have these beautiful campuses, nobody will have anything like this ${ }^{1}$. Ce projet semble l'incarnation même de la transition du territoire vers le capitalisme cognitif-culturel.

Fig. 7 : L'ancienne Gare Centrale

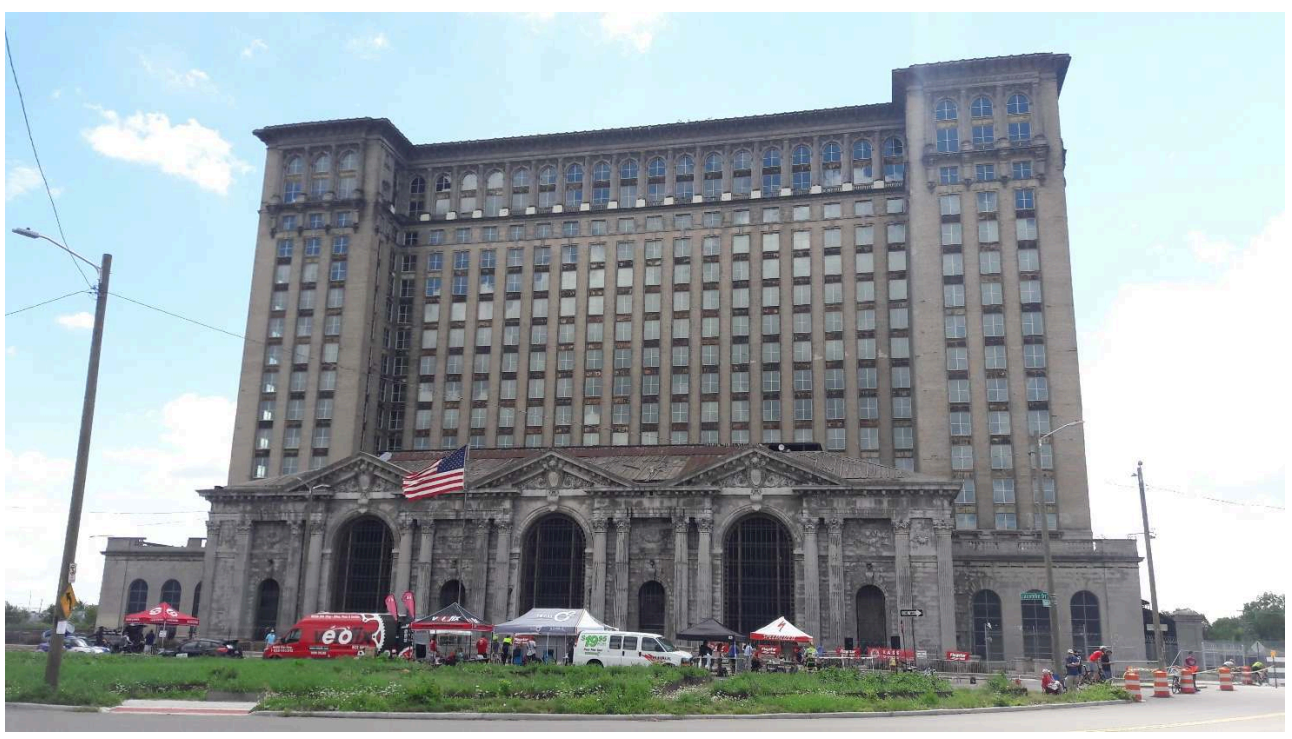

Photo de l'auteur (mai 2016)

\section{Conclusion}

Au terme de sa reconversion territoriale entamée dès les années 1980 mais qui s'est cristallisée au tournant des années 2010, Detroit a connu d'importantes reconfigurations sociales et spatiales qui vont dans le sens d'une fragmentation accrue de son espace métropolitain. Auparavant séparée en deux espaces distincts, la métropole semble désormais fragmentée en trois espaces: le GD gentrifié, «l'inner 
city " et les "suburbs». Ces reconfigurations ont aussi eu pour conséquence une revalorisation de la ville, à la fois sur le plan symbolique de son image et sur le plan spatial de ses valeurs foncières.

La culture a joué un rôle important dans ces transformations. Remodelée par les " creativity narratives ", elle s'apparente à un mythe d'action publique destiné à mettre en récit et légitimer cette dernière. À cet égard, le glissement vers les formules de la créativité témoigne à nos yeux d'un degré supplémentaire dans l'instrumentalisation de la culture au service de la performance économique. Ce phénomène n'est pas nouveau mais semble aller s'intensifiant avec le déploiement du régime du capitalisme cognitif-culturel.

\section{BIBLIOGRAPHIE}

Ambrosino, Charles et Andres, Lauren, « Régénération et mutabilité urbaine : un regard francobritannique », in L'économie culturelle et ses territoires, 305-17. Toulouse : Presses Universitaires du Mirail, 2008, p.305-17.

Béal, Vincent, Cary, Paul, Fol, Sylvie et Rousseau, Max, « Introduction », Géographie, économie, société, vol. 21, n 1, 2019, p. 5-22.

Benabdellah, Alia, « Black to Detroit. Aux origines médiatiques de la techno », Réseaux, $\mathrm{n}^{\circ} 223$, 2020, p. 129-155.

Boltanski, Luc et Esquerre, Arnaud, Enrichissement : une critique de la marchandise, Paris, Gallimard, 2017.

Bouquillion, Philippe (éd.), Creative economy, creative industries : des notions à traduire, Saint-Denis, Presses universitaires de Vincennes, 2012.

Bouquillion, Philippe, Miège, Bernard et Moeglin Pierre, L'industrialisation des biens symboliques. Les industries créatives en regard des industries culturelles, Grenoble, Presses universitaires de Grenoble, 2013.

Bouquillion, Philippe, «Industries du contenu et industries de la communication. Contribution à une déconstruction de la notion de créativité ", Les Enjeux de l'information et de la communication, $\mathrm{n}^{\circ} 16 / 3 \mathrm{~B}, 2015$, p.18-26.

Briche, Henri, « "Urbanisme d'austérité" et marginalisation des acteurs publics d'une ville en déclin : le cas de la rénovation urbaine à Detroit », Métropoles, (18), 2016. [En ligne]

Cooke, Philip et Lazzeretti, Luciana (éd.), Creative cities, cultural clusters and local economic development, Cheltenham, Edward Elgar, 2008.

Côté, Jean-François et Pugliese, Maud, « L'exposition Espace mobile à la Galerie VOX, de Montréal : régénération esthétique de l'espace public aux marges du politique », Études de communication, (31), 2008, p. 37-58.

Desage, Fabien et Godard, Jérôme, « Désenchantement idéologique et réenchantement mythique des politiques locales ", Revue française de science politique, vol. 55, 2005, p. 633-661. 
Detroit Future City, « 2012 Detroit Strategic Framework Plan », Detroit, Inland Press, 2012.

Detroit Renaissance, « Road to Renaissance. Final Recommendations », Detroit, New Economy Strategies, 2006.

Detroit Renaissance, « Detroit Strategic Planning Project », Detroit, 1987.

Doucet, Brian et Smit, Edske, « Building an urban "renaissance": fragmented services and the production of inequality in Greater Downtown Detroit », Journal of Housing and the Built Environment, 31, 2016, p. 635-657.

Doucet, Brian, « Deconstructing Dominant Narratives of Urban Failure and Gentrification in a Racially Unjust City: The Case of Detroit ", Tijdschdrift voor Economische en Sociale Geografie, vol. $111, \mathrm{n}^{\circ} 4,2020$, p. 634-651.

Florida, Richard, The rise of the creative class: and how it's transforming work, leisure, community and everyday life, New York, Basic Books, 2002.

Garnham, Nicholas, « From cultural to creative industries », International Journal of Cultural Policy, 11 (1), 2005, p. 15-29.

Gravari-Barbas, Maria et Ripoll, Fabrice, « Introduction : De l'appropriation à la valorisation, et retour », Norois. Environnement, aménagement, société, n²17, 2010, p. 7-12.

Gravari-Barbas, Maria (éd.), Aménager la ville par la culture et le tourisme, Paris, Éditions Le Moniteur, 2013.

Hall, Leda et Hall, Melvin, F. « Detroit's urban regime: composition and consequences », MidAmerican Review of Sociology, vol. 27, n², 1993, p. 19-37.

Houllier-Guibert, Charles-Edouard, « Gérer l'image des territoires avec le marketing territorial : émergence d'un « place branding » francophone? », Gestion et management public, 6/1 (3), 2017, p. 6-7.

Hudson Webber Foundation, « 7.2 square miles Report. Second Edition », Detroit, 2015.

Karpik, Lucien, L'économie des singularités, Paris, Gallimard, 2007.

Kinder, Kimberly, DIY Detroit: Making Do in a City without Services, Minneapolis: University of Minnesota Press, 2016.

Kresge Foundation, «Creative Vitality in Detroit. The Detroit Cultural Mapping Project », Detroit, Millier Dickinson Blais, 2012.

Krieg-Planque, Alice, « La formule développement durable : un opérateur de neutralisation de la conflictualité », Langage et société, n 134, 2010, p. 5-29.

Mah, Julie, « Gentrification-Induced Displacement in Detroit, Michigan: An Analysis of Evictions ", Housing Policy Debate, 2020. [En ligne]

Markusen, Ann et Gadwa, Anne, « Creative Placemaking Paper », Washington D. C., Mayors' Institute on City Design and the National Endowment for the Arts, 2010.

Menger, Pierre-Michel, Portrait de l'artiste en travailleur, Paris, Seuil-La République des Idées, 2002.

Mortelette, Camille, Reconversion d'anciens sites miniers en lieux culturels. Enjeux territoriaux et appropriation dans le Bassin minier du Nord-Pas-de-Calais, thèse de doctorat en géographie, Université d'Artois, 2019

Moulier-Boutang, Yann, « Art et capitalisme cognitif », L'Observatoire, Hors-série 3, 2010, p. 43-48. 
Neill, William, J. V., « Lipstick on the Gorilla: The Failure of Image-led Planning in Coleman Young's Detroit », International Journal of Urban and Regional Research, 19(4), 1995, p. 639-653. Noyer, Jacques, Raoul, Bruno et Pailliart, Isabelle (dir.), Médias et territoire. L'espace public entre communication et imaginaire territorial, Villeneuve d'Ascq, Presses du Septentrion, 2013.

Orr, Marion et Stoker, Gerry, « Urban regimes and leadership in Detroit », Urban Affairs Quarterly, vol. 30, n 1, 1994, p. 48-73.

Paddeu, Flaminia, « Faire face à la crise économique à Detroit : les pratiques alternatives au service d'une résilience urbaine? ", L'Information géographique, 76 (4), 2012, p. 119-39.

Paddeu, Flaminia, De la crise urbaine à la réappropriation du territoire : Mobilisations civiques pour la justice environnementale et alimentaire dans les quartiers défavorisés de Detroit et du Bronx à New York, Thèse de doctorat en géographie, Université Paris-Sorbonne, 2015.

Piore, Michael et Sabel, Charles, Les Chemins de la prospérité : De la production de masse à la spécialisation souple. Paris, Hachette, 1984/1989.

Reitel, Bernard et Moullé, François, « La resémantisation de la ligne frontière dans des régions métropolitaines transfrontalières : le Jardin des 2 Rives à Strasbourg et la place Jacques Delors à Lille », Belgeo, 2, 2015. [En ligne]

Renoir, Simon, Le tournant créatif à Detroit : enjeux croisés de la structuration des industries créatives et de la régénération urbaine, thèse de doctorat en Sciences de l'information et de la communication, Université Paris 13, 2018.

Renoir, Simon, « Detroit : le tournant créatif autour du design ? », Communication, 36/1, 2019. [En ligne]

Safransky, Sara, « Greening the urban frontier: Race, property, and resettlement in Detroit », Geoforum, 56, 2014, p.237-48.

Scott, Allen J., « Beyond the Creative City: Cognitive-Cultural Capitalism and the New Urbanism ", Regional Studies, 48 (4): 2014a, p. 565-78.

Scott, Allen J., « Villes et régions du capitalisme cognitif et culturel », L'espace géographique, 43, 2014 b, p. 215-226.

Seymour, Eric et Akers, Joshua, « Building the eviction economy: speculation, precarity and eviction in Detroit », Urban Affairs Review, 2019, p. 1-35.

Slotkin, Richard, The fatal environment: the myth of the frontier in the age of industrialization, 1800-1890, Norman, University of Oklahoma Press, 1998

Smith, Neil, The New Urban Frontier: Gentrification and the Revanchist City, London, New York: Routledge, 1996.

Sugrue, Thomas, J., The origins of the urban crisis: race and inequality in postwar Detroit, Princeton (N.J.), Princeton University Press, 1996.

Thomas, June, M. et Bekkering, Henco, Mapping Detroit: Land, Community, and Shaping a City, Detroit: Wayne State University Press, 2015.

Tremblay, Gaëtan, « Industries culturelles, économie créative et société de l'information », Global Media Journal, Edition Canadienne, Vol. 1, $\mathrm{n}^{\circ}$ 1, 2008, p. 65-88.

Vivant, Elsa, Qu'est-ce que la ville créative ? Paris, Presses universitaires de France, 2009

Wacquant, Loïc, Parias urbains. Ghetto, banlieues, État, Paris, La Découverte, 2005. 
Zitcer, Andrew, « Making Up Creative Placemaking », Journal of Planning Education and Research, 1-11, 2018. [En ligne]

Zukin, Sharon, et al. « New Retail Capital and Neighborhood Change: Boutiques and Gentrification in New York City », City \& Community, 8(1), 2009, p. 47-64

\section{NOTES}

1.

«La recherche de nouveaux talents fait l'objet une lutte sans merci et notre nouveau campus fera pâlir d'envie les plus grandes entreprises du numérique ». Source :

https://eu.freep.com/story/money/cars/ford/2020/08/05/detroit-train-station-renovationford/5548057002/ , consulté le 29/11/2020.

\section{RÉSUMÉS}

Depuis une dizaine d'années, la ville de Detroit aux États-Unis est engagée dans une reconversion territoriale de grande ampleur. Le registre culturel y joue un rôle central et, comme presque partout ailleurs, il a été refaçonné au cours de la période par les discours et les politiques sur la créativité, et sur les industries et les villes dites «créatives ». Cette transformation des discours et des politiques par la référence à la créativité est d'abord resituée dans le contexte plus large d'une transition vers un régime d'accumulation cognitif-culturel du capitalisme. Ensuite, cet article propose de décrire et d'expliquer le rôle de la culture et des industries créatives dans les transformations sociales et spatiales en cours à Detroit. Un premier point traite de la référence à la créativité comme point commun qui a facilité la convergence des divers projets de revitalisation urbaine depuis la fin des années 2000 à Detroit. Un deuxième point examine le rôle de la culture et des industries créatives dans le changement d'image de la ville, ainsi que dans diverses opérations de production de l'espace. L'ensemble aboutit à la constitution d'un nouvel espace, le «Greater Downtown », qui semble désormais séparé du reste de la ville.

For the past ten years, the city of Detroit in the United States has gone through a large-scale territorial reconversion. Culture has played a pivotal role in the process and, as almost everywhere else, it has been reshaped by discourses and policies on creativity and so-called "creative" industries and cities. This article resituates the transformation of discourse and policy within the broader framework of the transition towards cognitive-cultural capitalism. Next, it proposes to describe and explain the role of culture and creative industries in the recent social and spatial transformations taking place in Detroit. First, it focuses on the creativity narratives that have permeated local discourse since the end of the 2000s in Detroit and facilitated the convergence of various urban revitalization projects. Second, it examines the role of culture and the creative industries in changing the image of the city and prompting different modes of spatial production. The result is the creation of a new space, "Greater Downtown Detroit" which now seems to be separated from the rest of the city. 
En la última década, la ciudad de Detroit ha atravesado profundos cambios socio-espaciales y económicos. El registro cultural desempeña aquí un papel central y, como en casi todas partes, ha sido remodelado durante el período por los discursos y las políticas sobre la creatividad y sobre las llamadas industrias y ciudades "creativas". Esta transformación de los discursos y de las políticas por referencia a la creatividad se resitua primero en el contexto más amplio de una transición hacia un régimen de acumulación cognitivo-cultural del capitalismo. A continuación, este artículo busca describir y explicar el rol de la cultura y las industrias creativas en los recientes cambios sociales y espaciales de Detroit. Un primer punto se refiere a la referencia a la creatividad como punto común que ha facilitado la convergencia de los diversos proyectos de revitalización urbana desde finales de los años 2000 en Detroit. Un segundo punto examina el papel de la cultura y de las industrias creativas en el cambio de imagen de la ciudad, así como en diversas operaciones de producción espacial. El resultado es la creación de un nuevo espacio, el «Greater Downtown», que ahora parece estar separado del resto de la ciudad.

\section{INDEX}

Mots-clés : Detroit, ville créative, capitalisme cognitif-culturel, attractivité territoriale, reconversion créative

Palabras claves : Detroit, ciudad creativa, capitalismo cognitivo-cultural, atractivo territorial, reconversión creativa

Keywords : Detroit, creative city, cognitive-cultural capitalism, city branding, creative reuse

\section{AUTEUR}

\section{SIMON RENOIR}

Docteur qualifié en sciences de l'information et de la communication. Enseignant contractuel Université de Lille - membre associé du LabSIC, UFR Deccid - Campus Pont de Bois - Rue du Barreau - BP 60149 - 59653 - Villeneuve d'Ascq Cedex simon.renoir@gmail.com 